\title{
Slip Complexity in a Crustal-Plane Model of an Earthquake Fault
}

\author{
Christopher R. Myers, ${ }^{1}$ Bruce E. Shaw, ${ }^{2}$ and J. S. Langer ${ }^{3}$ \\ ${ }^{1}$ Cornell Theory Center, Cornell University, Ithaca, New York 14853 \\ ${ }^{2}$ Lamont-Doherty Earth Observatory, Columbia University, Palisades, New York 10964 \\ ${ }^{3}$ Department of Physics, University of California Santa Barbara, Santa Barbara, California 93106
}

(Received 30 June 1995)

\begin{abstract}
We study numerically the behavior of a two-dimensional elastic plate (a crustal plane) that terminates along one of its edges at a fault boundary. Slip-weakening friction at the boundary, inertial dynamics in the bulk, and uniform slow loading via elastic coupling to a substrate combine to produce a complex, deterministically chaotic sequence of slipping events. We observe a power-law distribution of small events and an excess of large events. For the small events, the moments scale with rupture length in a manner that is consistent with seismological observations. For the large events, rupture occurs in the form of narrow propagating pulses. [S0031-9007(96)00703-X]
\end{abstract}

PACS numbers: 91.30.Bi, 03.40.Dz, 05.45.+b, 64.60.Ht

The discovery of dynamic complexity in the uniform, one-dimensional Burridge-Knopoff (BK) model of an earthquake fault $[1,2]$ has brought new urgency to some questions about models of seismic sources. Perhaps the most pressing of these questions concerns the role of elasticity in the crustal plane-an ingredient that is necessarily missing in any one-dimensional model but which must be important for an understanding of the dynamics of slipping events. Off-fault elasticity is relevant to many features of real earthquake faults such as stress concentrations at rupture fronts, long-range elastic interactions, and seismic radiation.

Previous studies [3-7] indicate the following: The completely uniform, one-dimensional BK model, with velocity-weakening stick-slip friction, is a deterministically chaotic dynamical system that exhibits a broad range of earthquake-like events. The frequency-magnitude distribution for these events includes a scaling region of small localized events that is qualitatively similar to a Gutenberg-Richter (GR) law [8], and a region of large delocalized events whose frequency exceeds that of the extrapolated GR law and which account for most of the moment release. The large events propagate along the fault at roughly the sound speed in the form of "Heaton pulses" [9]. In order to be well posed mathematically, the model requires a cutoff or an ad hoc mechanism for initiating rupture at very small length scales. The questions of whether such mechanisms imply inherent discreteness of these models and whether that discreteness, in turn, implies the need for small-scale heterogeneity in realistic fault models are beyond the scope of this investigation. The important point is that the large-scale properties - complexity of large events and existence of a GR regime-are independent of the discretization length or the heterogeneity scale, and therefore appear to be robust features of this class of models.

Our purpose in the investigations reported here has been to test the above features of the one-dimensional BK models in a two-dimensional model that includes elasticity in the crustal plane. Like the one-dimensional models, our two-dimensional model is still a caricature of the physically realistic situation, but it brings us a large step closer to an understanding of the dynamic behavior of this class of systems. It remains unrealistic in at least two respects, both of which are dictated by computational feasibility. First, our crustal plane is an elastic plate that moves only normal to itself and is coupled elastically to a rigid substrate. In this way, we obtain an essential simplification of our equations of motion and retain a very important dynamic time scale associated with coupling between the top and bottom of the crust; but we lose the long-range behavior of true elasticity for very slow motions. Because we are interested primarily in source dynamics, we find this to be an acceptable compromise. Second, as in the one-dimensional models, we use an overly simplified mechanism for producing a stress drop at the initiation of rupture. We believe that this is an adequate approximation for an ordinary fracture on a smooth fault with a nucleation length roughly the same as our mesh size, but we cannot prove this without very extensive computation. (For an opposite point of view, see [10].) Again, we emphasize that this length scale appears to be an irrelevant parameter insofar as the observable, large-scale properties are concerned. Our main conclusions are that the crucial features listed in the preceding paragraph are indeed preserved in the new model, and that we are discovering a substantial degree of universality in these systems.

Our elastic plate occupies the $y>0$ half of the $(x, y)$ plane (the "crustal plane") and terminates at a fault on the $x$ axis (see Fig. 1). It is easiest to visualize the fault [the $(x, z)$ plane] as moving downward at the loading speed $\nu$ with respect to the plate when it is stuck, and exerting tractions on the edge of the plate via a stick-slip friction force. In a rough sense, we are modeling a vertical dip-slip fault. Throughout the plate, the dimensionless equation of motion for the displacement field $U(x, y, t)$ is a massive wave equation: 


$$
\frac{\partial^{2} U}{\partial t^{2}}=\frac{\partial^{2} U}{\partial x^{2}}+\frac{\partial^{2} U}{\partial y^{2}}-U+\nu t .
$$

Here, $U$ is measured in units of a slipping distance (of order meters) that is determined by the coupling to the substrato and the m.ximum drop in friction during klip. The loading rate $\nu$ is measured in units of the corresponding slipping speod (of order $\mathrm{m} / \mathrm{sec}$ ); thus, $\nu \ll$ 1. The position variables $x, y$ are expressed in units of a length (of order $10 \mathrm{~km}$ ) that we identify roughly as the thiclness of the seismogenic zone, i.e., the distance between the plate and the anbstrate. Acrordingly, onr umit of $t$ is the time taken for a sound wave to traverse this distance (of order sec).

The forces on the right-hend side of (1) are the twodimensional olastic strain pradients and the coupling to the moving substrate, $\nu t-U$. The latter term produces an unrealistic exponential decay of atatic strain gradients, but without it we would be missing the restoring force that produces the characteriatic period for large, unifomm crustal motions. In a fully threo-dimensional model with tensor mather than sealar elagticity, this period would appear as a low-frequenoy cutoff for Rayleigh waves on the foult plano.

To complete the model, we write the boundary condition at $y=0$ in the fom

$$
\partial U /\left.\partial y\right|_{y \rightarrow 0}=\Phi,
$$

where $\$$ is the rtict-olip firiction that provides the traction on the fant surface. Here we depant from orr previous practice and wse a slip-weakening rather than a velocitywerkening version of the friction law. One reason for doing this is that, in our opinion, the alip-weakening model is at least a partially coirect representation of the failure mechanism that cocurs on real faults. In a scensinio proposed by Sibson [11], frictional heeting raises the temperature and preasure of pore fluids, thereby reducing the effeotive normal atress and friction. This scenario leads to slip-weakening friction when hest dissipation is slow compinged to the rupture time scale $[12,13]$, and to velocity-

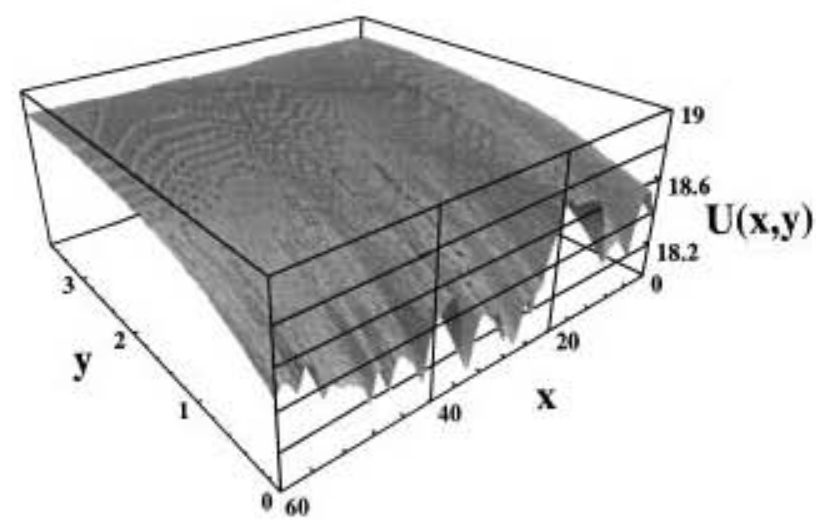

FIG. 1. The di.placement field $\boldsymbol{U}(\boldsymbol{x}, \boldsymbol{y})$ in $\mathrm{em}$ example of a fully stuxk configmintion. The variable $x$ is the distance along the fialt, and $y$ is the dinterose perpendivoler to the finlt. The fault boundary is the $x$ eds, elcong which the frictional stresess ans epplied. weakening friction when heat dissiption is relatively fast [13]. Volume expansion provides a mochanism for disaipation of pore preasure on the rupture time scale [14], a time scale intermediate between these two extremes. This suggests thet Bome mixture of slip-and velocity-wealening effects is relevint to earthquakes. We have examined both types of friction laws in this two-dimensional model and have found basically the same behavior in both cases. A geound resson for considering only the slip weskening in this discussion is that, as orplained below, it is methematically better behrved in the continum limit.

The form of slip-weakening friction that we use is

$$
\Phi= \begin{cases}{\left[-\infty, \phi\left(S-S_{0}\right)\right]} & \partial S / \partial t=0, \\ \phi\left(S-S_{0}\right)-\delta(t) & \partial S / \partial t>0,\end{cases}
$$

with

$$
\phi\left(s-s_{0}\right)=\left[1+\alpha_{s}\left(s-s_{0}\right)\right]^{-1} .
$$

Here, the function $S(x, t)=U(x, 0, t)$ denotes the displacement of the crust along the finlth $s_{0}(x)=$ $U\left[x, 0, t_{0}(x)\right]$ is the value of $S$ at the beginning of an event, and $t_{0}(x)$ is the time when slip starts at the point $x$. Equation (3) speoifies the atick-1lip nature of the friotion; it resists motion up to a threshald and decreases continitongly once slipping tharta. Note that $S(x)-S_{0}(x)$ is the total slip at $x$ starting from the beginning of an event, as is consistent with the relatively slow rate of heat dissipation in our phyzical picture of alip weakening. In a complex event, the material at a point $x$ may slip and rectick mone than once, but $\phi$ continues to decrease throughout this motion according to (3). Once the event is over, the fiult reheals, and the slipping threahold is reset to $\phi(0)=1$ evarywhere.

The term $\partial(t)$ is our special approximation for the rapid but contimuous drop in strongth that oecurrs when a material changes from sticking to slipping. In this work, wo heve used

$$
\delta(t)= \begin{cases}\sigma\left(t-t_{2}\right) / \tau & \left(t-t_{2}\right) / r<1, \\ \sigma & \left(t-t_{*}\right) / \tau \geq 1,\end{cases}
$$

60 that $\partial$ increases linearly with time once the frult becomes unstuck, up to a maximum value $\sigma$ over a time scale $\tau_{1}$ and is reset to zero when the fanlt resticks. The time $t_{2}$ is measured from the last unsticking and, unlike $S_{0}$, is reset during an event if the frult resticks and then slips again. This diffecence aries from our physical picture of the two terms, with $s-S_{0}$ arising from the bnilt up beeting effocts, and $\bar{\sigma}$ arising fiom transitions from sticking to alipping. When $\&$ depends only on time, as in (5), and is amall compared to the change in $\phi$ dos to slip weakening, the large events are found to be independent of $\sigma$. We also observe that, when we take $\tau$ small compared to the event timo scale, the stress drops in the small events seale with $\sigma$ and are independent of $\tau$.

There are two overall symmetrie in the equations which allow subtraction of a constant from $\$$ and ovexall multiplication by mother constant. These symmetries 
have been used to set to unity both the maximum sticking friction and the maximum drop in friction due to slip weakening. The reduction of friction $\phi$ with slip $S-S_{0}$ in (4) is the source of the crucial instability that leads to slip complexity, and the slip-weakening parameter $\alpha_{s}$ determines the strength of this instability. For $\alpha_{s}$ large compared to unity we see a generic complex behavior. We have used $\alpha_{s}=3$ throughout the computations described here. The exact functional form of $\phi$ also appears to be unimportant.

In the velocity-weakening case, the slip $S-S_{0}$ in (4) would be replaced by the slip rate $\partial S / \partial t$, and $\alpha_{s}$ would be replaced by, say, $\alpha_{v}$. The velocity-weakening model as defined in this way fails to have a well-defined continuum limit. The difficulty can be understood by examining the linear growth rates for sinusoidal deformations on the fault surface during slipping in the frictional weakening regime. For a perturbation of the form $U \sim \exp (i k x-$ $\kappa y+\Omega t$ ), we find that

$$
\kappa=\alpha_{s}, \quad \Omega=\sqrt{\alpha_{s}^{2}-k^{2}-1}
$$

for slip weakening, and

$$
\kappa=\alpha_{v} \Omega, \quad \Omega=\sqrt{\left(k^{2}+1\right) /\left(\alpha_{v}^{2}-1\right)}
$$

for velocity weakening. There is no apparent problem in going to a continuum limit for the case of slip-weakening friction; the smallest wavelengths remain marginally stable. In contrast, for velocity weakening, the smallest wavelengths are strongly unstable. A viscous force of the form $\eta \partial^{3} S / \partial^{2} x \partial t$ cures this difficulty in the one-dimensional BK model $[5,6]$ and is also useful in a related two-dimensional model of ordinary fracture [15].

In our numerical integrations, we have used a finitedifference scheme with a variety of grid spacings. We have performed our numerical integrations using a finite, rectangular grid of physical size $L_{x}$ by $L_{y}$ and grid spacings $\delta x$ and $\delta y$. We impose periodic boundary conditions in the $x$ direction (along the fault) and a zero-normal-derivative (Neumann) condition along the boundary at $y=L_{y}$. Because the plate is necessarily finite in the $y$ direction, we need to minimize the extent to which elastic waves reflect back upon the fault from the system's outer edge at $y=L_{y}$. To accomplish this, we have added a layer of viscous damping to the equation of motion (1) near the outer edge of the form $\eta(y) \nabla^{2} \partial U / \partial t$, with $\eta(y)$ rising smoothly from zero at $y=3 L_{y} / 4$ and saturating at a value of 0.5 at the outer boundary. Our finite-difference scheme steps forward in time using an explicit Euler method which is first-order accurate in the time step $\delta t$. Spatial derivatives, both in the bulk and on the boundary, are accurate to $O\left((\delta x)^{2},(\delta y)^{2}\right)$. In all of the calculations reported here we have used $\tau=0.2$ and $\sigma=0.03$. (This value of $\sigma$ may be unrealistically small but is convenient for clarifying the distinction between large and small events in these limited simulations.)

Beginning from an arbitrary nonuniform initial configuration, our system evolves into a statistically steady state with a rough configuration and a wide range of event sizes. Figure 1 shows a typical displacement field $U$ in a fully stuck configuration. The displacements are inhomogeneously locked by the friction on the fault, and decay exponentially into the bulk over length scales of order unity.

Figure 2 shows a sequence of stuck configurations at the fault boundary as the plate moves forward. Note that there are many small events (most of which are not visible) and fewer large ones, but almost all of the forward motion occurs in the large events. One way to characterize this complexity is to look at the differential distribution $R$ of event magnitudes $\mu=\log _{10} M$, which is shown in Fig. 3. The moment $M$ is the total slip on the fault during an event:

$$
M=\int\left[S_{f}(x)-S_{0}(x)\right] d x,
$$

where $S_{0}$ and $S_{f}$ are the initial and final configurations. Just as in the one-dimensional cases, $\log _{10} R(\mu)$ has a straight-line scaling or power-law region with negative slope $b \approx 1$ for small events, and a distinct peak for the large events. The value of $b$ for the small events is a robust feature of our dynamic models, holding for both slip and velocity weakening, and in lower dimensions as well. (However, the medium events are exponentially suppressed in the one-dimensional slip-weakening model, perhaps because the dynamic instability is weakest in that case.) We also show in Fig. 3 that these distributions are insensitive to changes in the grid spacings. Of course, as we decrease $\delta x$, we add new small-scale events to the bottom of the distribution. Aside from this effect, the curves lie on top of one another to within our statistical uncertainty. We have also checked that there is no appreciable dependence of these distributions on the dimensions of the system, $L_{x}$ and $L_{y}$.

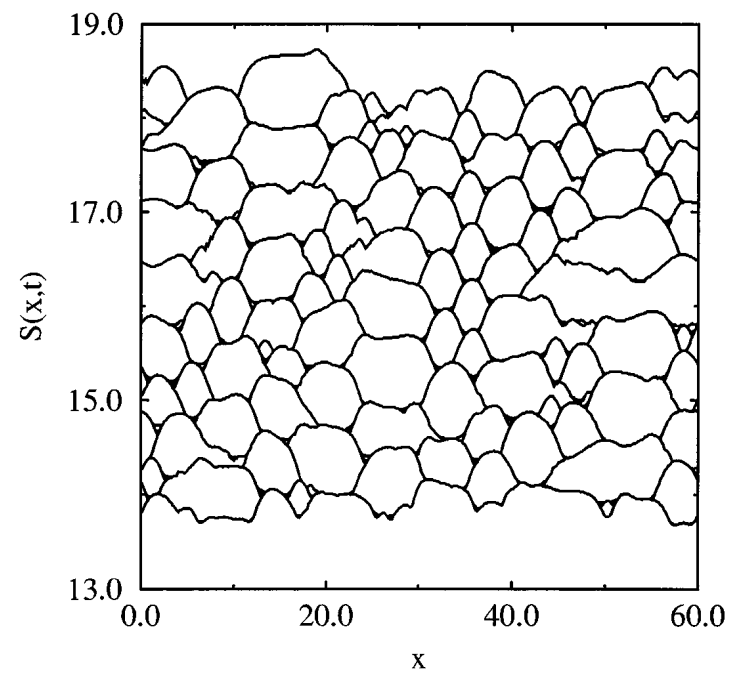

FIG. 2. A sequence of stuck configurations of the displacement at the fault boundary. The area between subsequent configurations is the moment $M$ of an event. The lattice parameters used in this figure are $\delta x=0.15, \delta y=0.075, L_{x}=60$, and $L_{y}=3.75$. 


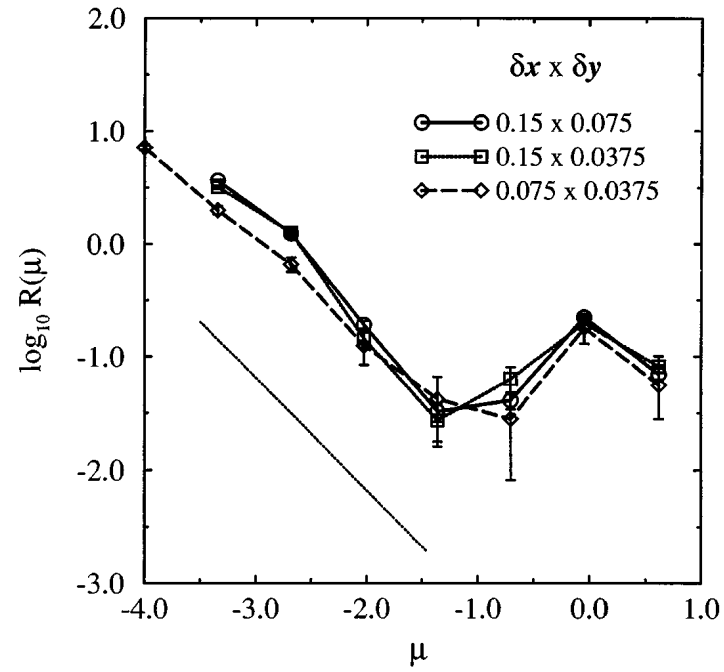

FIG. 3. The differential magnitude distribution and a demonstration showing that these results are insensitive to changes in the grid spacing. $R(\mu)$ is the number of events with magnitudes between $\mu$ and $\mu+d \mu$ per unit fault loading per unit fault length. The three curves differ only in their grid spacings as shown. $L_{x}=60$ and $L_{y}=3.75$ for all curves. Also plotted is a line of slope -1 .

One respect in which the two-dimensional model differs from the one-dimensional version is the correlation between the moment $M$ and the source dimension $\Delta$ (the size of the region that slips in an event). In the Earth, this correlation is well fit over a wide range of source dimensions by an assumption of constant stress drop, which implies that the average slip scales linearly with $\Delta$. Therefore, for our two-dimensional model, we expect $M \sim \Delta^{2}$. The dashed lines in Fig. 4 indicate that we see this behavior throughout the GR region, with $M \sim$ $\sigma \Delta^{2}$ for the small events. The average slip $M / \Delta$ continues to increase with slip-zone size $\Delta$ up to and beyond $\Delta=1$, which is our analog of the crust depth. A similar phenomenon has been reported for real earthquakes by Scholz [16]. For the very largest events in Fig. 4, we see $M \sim \Delta$, which means that the average slip becomes independent of slip-zone size. These are delocalized events in which slip occurs in the form of propagating, self-healing pulses which fit the scenario advocated by Heaton [9]. Analytic progress in understanding aspects of these pulses has been made recently by Langer and Tang [4], Myers and Langer [5], and Langer and Nakanishi [15]. Details of the present results will be reported elsewhere.

In conclusion, we have demonstrated that this twodimensional elastodynamic model with slip-weakening friction along a homogenous one-dimensional fault boundary produces slip complexity. We believe that these results support the case that inertial dynamics and frictional weakening are contributing in fundamental ways to earthquake complexity.

C.R.M. was supported by the NSF under grant ASC-9309833, and the Cornell Theory Center, which

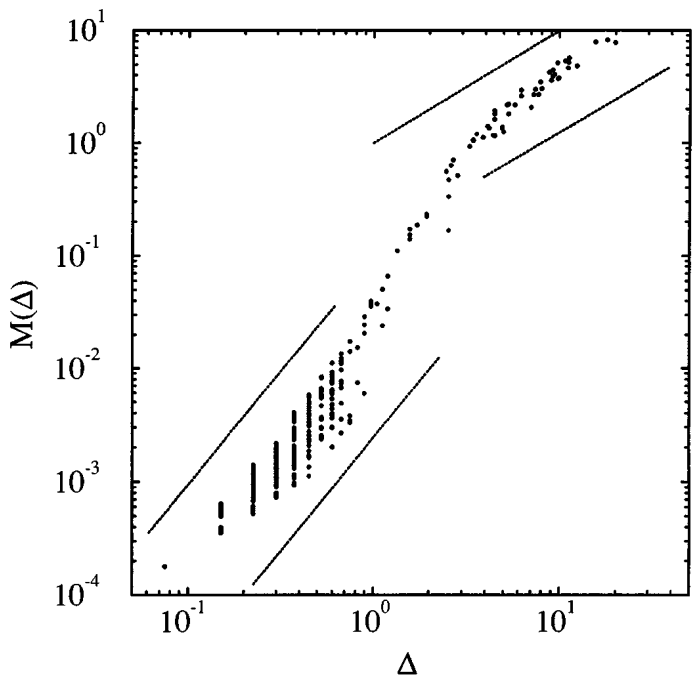

FIG. 4. The moment $M$ as a function of the slip-zone size $\Delta$. The dots indicate individual events. The lower two dashed lines have slope 2; the upper two lines have slope 1. $\sigma=0.03$ sets the stress drop of the small events.

receives major funding from the NSF and New York State. C.R.M. also acknowledges support from Los Alamos National Laboratory under an INCOR Collaborative Research Grant. B.E.S. was supported by NSF Grant 93-16513 and by SCEC Grant USC569934. J.S.L. was supported by DOE Grant DEFG03-84ER45108 and NSF Grant PHY89-04035; both C. R.M. and B.E.S. acknowledge support from the latter grants during the early stages of this project.

[1] R. Burridge and L. Knopoff, Bull. Seismol. Soc. Am. 57, 3411-3471 (1967).

[2] J.M. Carlson and J. S. Langer, Phys. Rev. A 40, 6470 (1989).

[3] J. M. Carlson, J. S. Langer, B. E. Shaw, and C. Tang, Phys. Rev. A 44, 884-897 (1991).

[4] J. S. Langer and C. Tang, Phys. Rev. Lett. 67, 1043 (1991).

[5] C.R. Myers and J. S. Langer, Phys. Rev. E 47, 3048 (1993).

[6] B.E. Shaw, Geophys. Res. Lett. 21, 1983 (1994).

[7] J.M. Carlson, J.S. Langer, and B.E. Shaw, Rev. Mod. Phys. 66, 657-670 (1994).

[8] B. Gutenberg and C.F. Richter, Seismicity of the Earth and Associated Phenomena (Princeton University, Princeton, NJ, 1954).

[9] T. H. Heaton, Phys. Earth Planet. Inter. 64, 1 (1990).

[10] J. R. Rice, J. Geophys. Res. 98, 9885 (1993).

[11] R. H. Sibson, Nature (London) 243, 66 (1973).

[12] A. Lachenbruch, J. Geophys. Res. 85, 6097 (1980).

[13] B. E. Shaw, J. Geophys. Res. 100, 18239 (1995).

[14] C. W. Mase and L. Smith, J. Geophys. Res. 92, 6249 (1987).

[15] J.S. Langer and H. Nakanishi, Phys. Rev. E 48, 439 (1993).

[16] C. H. Scholz, Bull. Seismol. Soc. Am. 72, 1-14 (1982). 